\title{
The Prototype Opto-mechanical System for the Fluorescence detector Array of Single-pixel Telescopes
}

Dusan Mandat ${ }^{*}$, , Miroslav Palatka ${ }^{a}$, Miroslav Pech ${ }^{a}$, Petr Schovanek ${ }^{a}$, Petr Travnicek $^{a}$, Libor Nozka ${ }^{b}$, Pavel Horvath $^{b}$, Miroslav Hrabovsky $^{b}$, Justin Albury ${ }^{c}$, Jose A. Bellido ${ }^{c}$, John Farmer ${ }^{d}$, Toshihiro Fujii ${ }^{e}$, Aygul Galimova $^{d}$, Max Malacari ${ }^{d}$, Ariel Matalon ${ }^{d}$, John N. Matthews ${ }^{f}$, Maria Merolle ${ }^{d}$, Xiaochen $\mathbf{N i}^{d}$, Paolo Privitera ${ }^{d}$, Stan B. Thomas ${ }^{f}$ (FAST Collaboration) (F $^{\ddagger}$

${ }^{a}$ Institute of Physics of the Academy of Sciences of the Czech Republic, Prague, Czech Republic

${ }^{b}$ Palacky University, RCPTM, Olomouc, Czech Republic

${ }^{c}$ Department of Physics, University of Adelaide, Adelaide, S.A., Australia

${ }^{d}$ Kavli Institute for Cosmological Physics, University of Chicago, Chicago, IL, USA

${ }^{e}$ Institute for Cosmic Ray Research, University of Tokyo, Kashiwa, Chiba, Japan

${ }^{f}$ High Energy Astrophysics Institute and Department of Physics and Astronomy, University of Utah, Salt Lake City, UT, USA

E-mail: mandat@fzu.cz

\begin{abstract}
We present the opto-mechanical design of a new generation fluorescence telescope for the detection of ultrahigh-energy cosmic rays (UHECRs). The Fluorescence detector Array of Singlepixel Telescopes (FAST) is a proposed low-cost, large-area, next-generation experiment for the detection of UHECRs via the atmospheric fluorescence technique. The telescope is of a simplified Schmidt design, suitable for a camera consisting of only a few large pixels. The telescope has a $1 \mathrm{~m}^{2}$ entrance aperture, and a field-of-view of $30^{\circ} \times 30^{\circ}$. We present the optical design of the prototype telescope as well as the mirror alignment and pointing calibration procedures. The prototype of the FAST telescope is installed at the Black Rock Mesa site of the Telescope Array Experiment.
\end{abstract}

35th International Cosmic Ray Conference - ICRC2017

10-20 July, 2017

Bexco, Busan, Korea

\footnotetext{
${ }^{*}$ Corresponding author.

$\dagger$ Presenter.

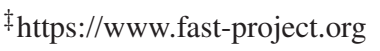




\section{Fluorescence detector Array of Single-pixel Telescopes (FAST)}

The Fluorescence detector Array of Single-pixel Telescopes (FAST) [1] is a design concept for a low-cost, ground-based fluorescence detector. A FAST telescope would consist of just four pixels covering a $30^{\circ} \times 30^{\circ}$ patch of the sky with a $\sim 1 \mathrm{~m}^{2}$ collecting area. Its low cost would facilitate deployment over a very large ground area, making it a viable candidate for a next-generation cosmic ray observatory. Such a design comes at the expense of low energy performance, as the signal-tonoise $(\mathrm{S} / \mathrm{N})$ ratio measured by a photomultiplier-tube (PMT) is proportional to $\sqrt{A / \Delta \Omega}$ [2], where $A$ is the light collecting area and $\Delta \Omega$ is the pixel solid angle, which is $\sim 15^{\circ}$ in the FAST design (compared with, for example, $A \sim 3 \mathrm{~m}^{2}$ and $\Delta \Omega \sim 1.5^{\circ}$ for the fluorescence telescopes of the Pierre Auger Observatory [3]). In addition, reconstruction of the geometry of an EAS with adequate resolution using data collected by a single FAST telescope is unlikely, as the coarse granularity of a $2 \times 2$ matrix of PMTs does not supply sufficient timing information to remove degeneracy in the determination of the shower axis. However, showers of sufficiently high energy would be observed by multiple FAST telescopes in an array, in which case timing information from the involved telescopes, along with the shape of the detected light pulse, could allow for reconstruction of the shower geometry with reasonable accuracy. An array of FAST telescopes would also be well suited as a complementary fluorescence detector to a sparse array of ground-based particle detectors, which could supply the shower geometry independently.

\section{FAST prototype optical design}

The prototype utilizes a large segmented mirror telescope of $1 \mathrm{~m}^{2}$ collecting area to focus light onto a camera consisting of four $200 \mathrm{~mm}$ diameter PMTs. The prototype has been installed in a dedicated building alongside the fluorescence telescopes at the Black Rock Mesa site of the Telescope Array Experiment (TA) [4], where the design is being tested. Two additional FAST telescopes will be installed at the same location in September 2017, covering a total of $90^{\circ}$ in azimuth. The deployment of additional FAST telescopes will allow for a three-fold increase in the prototype aperture, greatly increasing the number of showers observed in coincidence with the TA fluorescence telescopes. The primary design goal of an individual FAST telescope is to have an optical system with an effective collecting area of $\sim 1 \mathrm{~m}^{2}$ and a $\sim 30^{\circ} \times 30^{\circ}$ field-of-view which is capable of focusing atmospheric fluorescence light onto a matrix of several $200 \mathrm{~mm}$ PMTs. In addition, it is required to be low-cost, straightforward to maintain, and easy to transport and install. The low cost requirement makes it necessary to minimize the number of optical elements in the telescope. A Schmidt type optical design was adopted for the full-size FAST prototype. In a typical Schmidt telescope a corrector plate is placed at the entrance aperture (located at the mirror's radius of curvature, a distance of $2 f$, where $f$ is the focal length) to facilitate the control of off-axis aberrations: coma and astigmatism. Field curvature and spherical aberration are still present, although the former can be eliminated by placing a suitably curved detector in the image plane. The size of the optical point spread function (PSF), which describes the spatial distribution of light on the focal surface, is a function of the spherical aberration of the system, and is typically circular in shape for both on- and off-axis beams. 
The coarse granularity of the FAST camera, having only four PMTs each covering an angular field-of-view of $\sim 15^{\circ}$, allows the requirements on the size and shape of the telescope's PSF to be relaxed. The FAST prototype telescope therefore takes the form of a lensless Schmidt camera, as residual coma and astigmatism present due to the lack of a corrector plate does not affect the functionality of the telescope. The telescope mirror is reduced in size, and the distance between the mirror and the focal surface shortened relative to a regular Schmidt telescope, with the entrance aperture located closer to the focal surface. The dimensions of the FAST prototype telescope are shown in Fig. 1. An octagonal aperture of height $1.24 \mathrm{~m}$ is located at a distance of $1 \mathrm{~m}$ from a $1.6 \mathrm{~m}$ diameter spherical mirror. The design fulfills the basic FAST prototype requirements, with an effective collecting area of $1 \mathrm{~m}^{2}$ after accounting for the camera shadow, and a field-of-view of $30^{\circ} \times 30^{\circ}$. The size and the shape of the spot is of particular importance, and is shown in

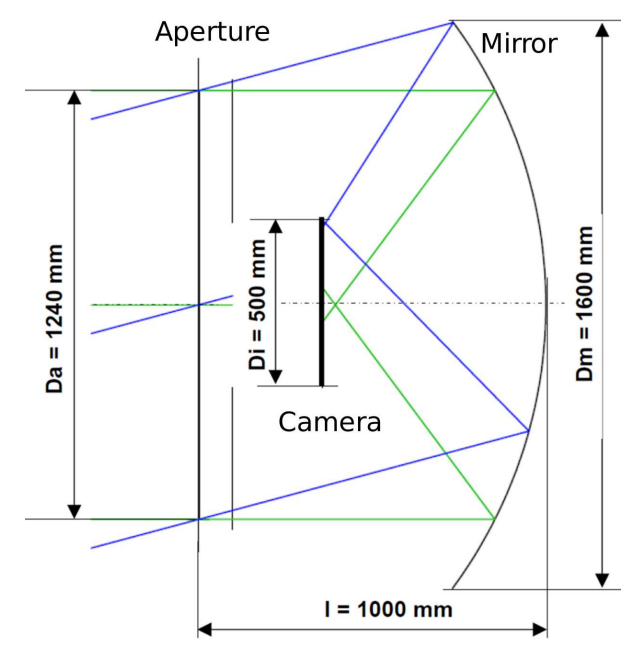

Figure 1: The dimensions of the FAST prototype telescope's optical system. $D_{\mathrm{a}}$ is the face-to-face size of the octagonal telescope aperture, $D_{\mathrm{i}}$ is the side length of the square camera box, $D_{\mathrm{m}}$ is the diameter of the primary mirror, and $l$ is the mirror-aperture distance.

Fig. 2 (note that this figure depicts the geometrical spot shape along with the intensity of the spot relative to the maximum). The top (bottom) row shows the spot shape for an on-axis (off-axis) optical beam as a function of the distance from the focal plane. The $300 \mathrm{~mm}$ scale represents the maximum diameter of the spot size, the PMT diameter is $200 \mathrm{~mm}$ and 4 of them will be installed in the camera located in a custom-built box at the focus of the optical system. The characteristic "star" shape of the optical spot is a result of the octagonal shape of the entrance aperture. The spot shape becomes circular in nature for positive defocusing of the telescope (the image plane moved closer to the mirror), with a central hole corresponding to the shadow of the camera box. In order to minimize the effect of the dead space between PMTs, a $25 \mathrm{~mm}$ negative defocusing was utilized in the prototype design. This serves to eliminate a complete loss of signal for on-axis optical beams where light is focused in the central point between all four PMTs. These simulations were performed assuming a single compact primary mirror, while the constructed FAST prototype uses a segmented primary mirror, complicating the shape of the optical spot. Nevertheless, these simple simulations accurately predict its size.

The FAST telescope design consists of a central circular mirror and 8 side mirrors, or "petals". 

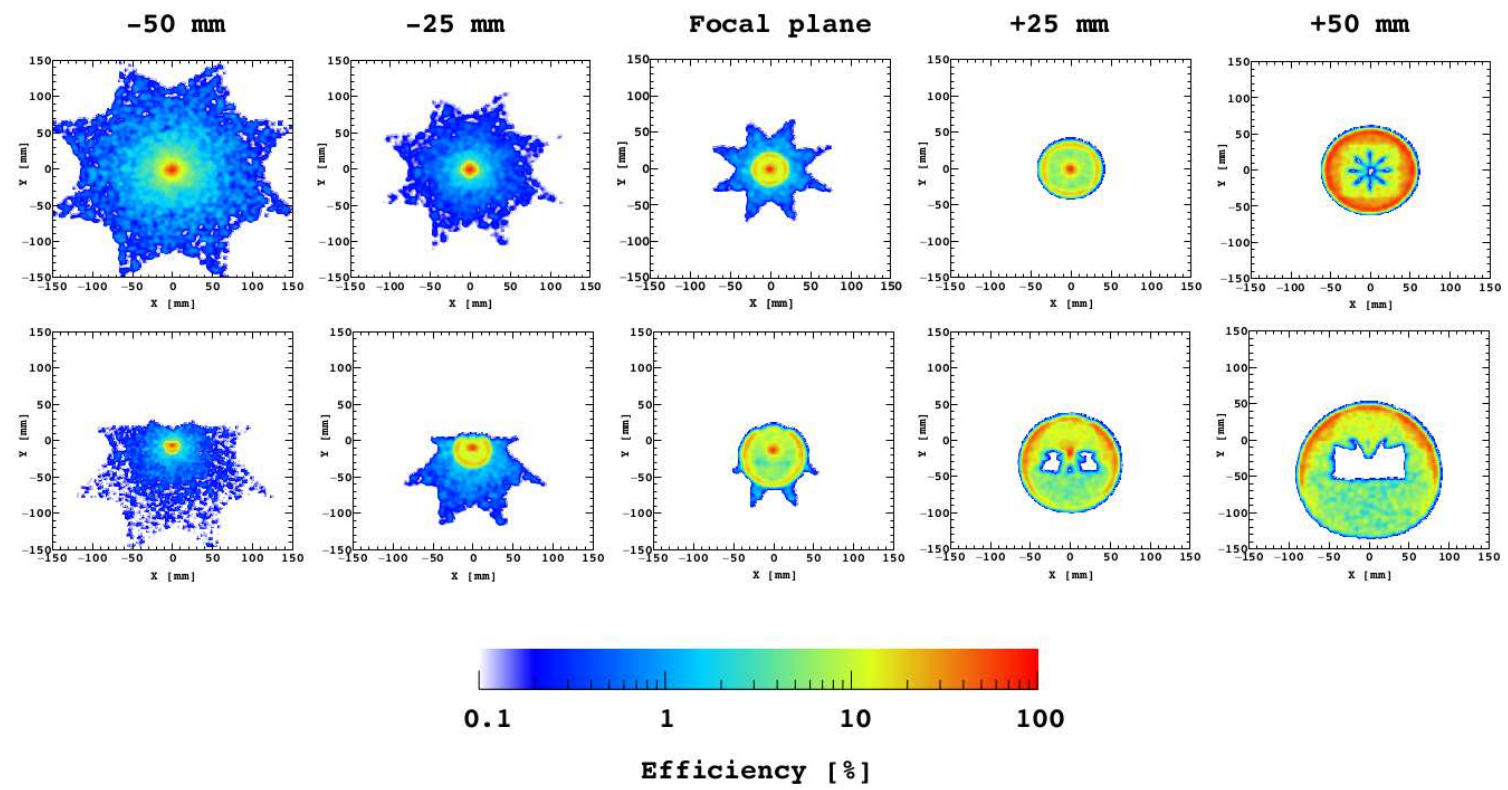

Figure 2: Geometrical spot diagrams from ray-tracing simulations of the FAST prototype optics. The spot diameters are shown for both on-axis (top) and off-axis (bottom) beams as a function of the distance from the focal plane (defocusing). Negative defocusing corresponds to moving the focal plane further from the mirror, and vice versa for positive defocusing. The color scale represents the relative intensity profile of the spots.

The diameter of the individual mirror segments was limited by the technology available in our laboratory. The mirrors are produced on site, in the Joint Laboratory of Optics of the Palacky University, and the Institute of Physics of the Academy of Sciences of the Czech Republic, from a custom-made substrate. The substrate is a borosilicate glass with good optical and mechanical quality. The reflective surface consists of vacuum coated $\mathrm{Al}$ and $\mathrm{SiO}_{2}$ layers. The typical spectral reflectance, filter transmission and total optical efficiency between $260 \mathrm{~nm}$ and $420 \mathrm{~nm}$ is shown in Fig. 3. The reflectivity is relatively constant over this wavelength range, with a maximum of $\sim 90 \%$ at $420 \mathrm{~nm}$, and a minimum of $\sim 75 \%$ at $260 \mathrm{~nm}$.

A UV band-pass filter is installed at the aperture of the telescope to reduce the exposure to night-sky background light. We use a ZWB3 filter manufactured by Shijiazhuang Zeyuan Optics. Its spectral transmission is shown in Fig. 3.

The telescope's mechanical support structure was built from commercially available aluminum profiles. This allows for straightforward assembly/disassembly, and easy packing and transport due to their light weight, while also providing an extremely stable and rigid platform for the FAST optical system to be mounted on. The mechanics consists of a primary mirror stand mounted with a single degree of freedom to facilitate adjustment of the telescope's elevation (the elevation can be set to discrete values of $0^{\circ}, 15^{\circ}, 30^{\circ}$ and $45^{\circ}$ above the horizon). The square camera box (side length $500 \mathrm{~mm}$ ), which holds four $200 \mathrm{~mm}$ PMTs, is mounted on a support structure connected to the perimeter of the mirror dish which also holds the octagonal filter aperture. The mirror stand contains 9 mirror mounts, each with 2 degrees of freedom to allow for mirror segment alignment. 


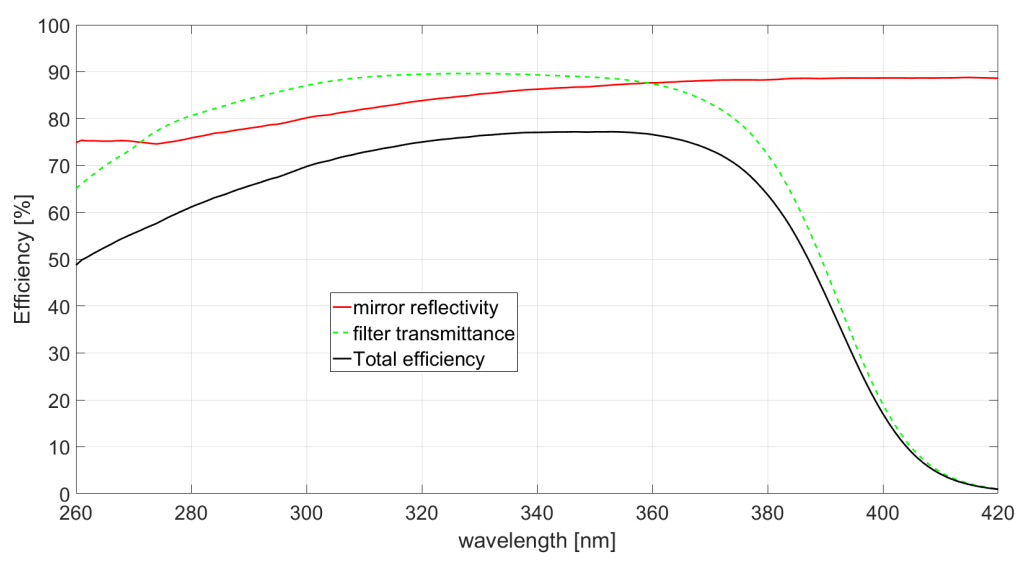

Figure 3: The typical spectral reflectance of the FAST mirror between $260 \mathrm{~nm}$ and $420 \mathrm{~nm}$, along with the spectral transmission of the UV band-pass filter. The resultant total optical efficiency is shown in black.

The whole mechanical construction, shown in Fig. 4, is covered with a shroud to protect the optical system from the surrounding environment.

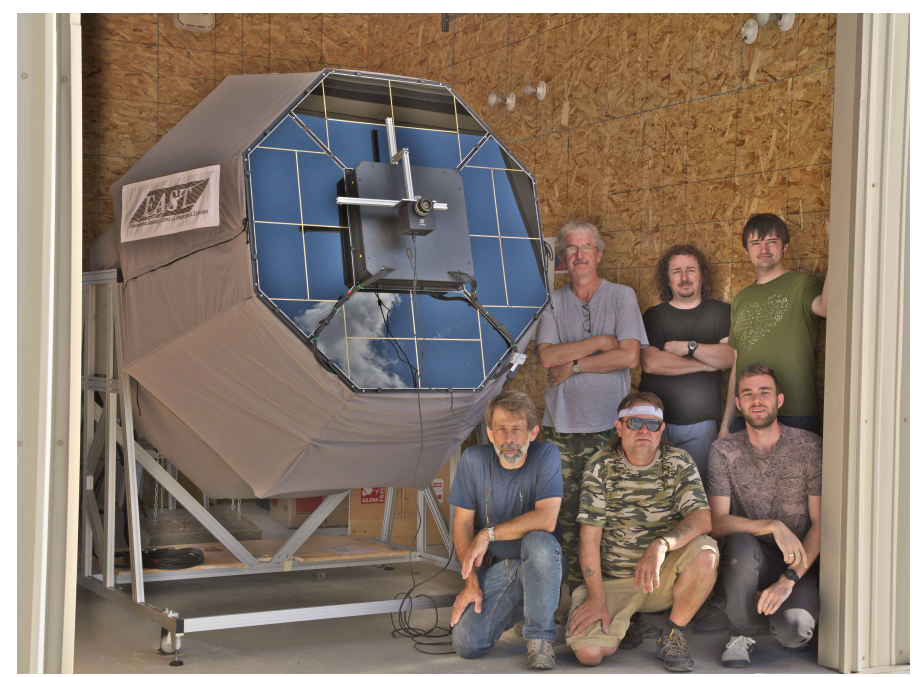

Figure 4: The complete mechanical structure. The mirror and the camera are protected from dust and aerosols by a shroud, which also acts as a shield for ambient light. The image was taken at the Black Rock Mesa site of TA following the installation.

\section{Mirror alignment}

The geometrical optical axis is defined by the line joining the center of the mirror dish to the center of the camera box. Once the geometrical axis of the FAST mirror dish is defined, all mirror segments must be aligned with respect to this axis. Each mirror is mounted on a support with two degrees of freedom for segment alignment, and a diode laser is mounted in the center of the mirror dish pointing along the optical axis. We use two light sources and two alignment techniques to 
accurately determine the final position of the mirrors. As the segmented mirror has a spherical shape, all mirror segments must point to the radius of curvature of the telescope. The light source is located along the optical axis of the telescope, at a distance of $2 f$ from the central mirror. The point-like source is reflected back to a screen mounted at the same location, and if the segments are well aligned the image of the light spot overlaps the light source. The second spot-like source is placed close to the telescope on the optical axis, at a distance $<f$. The light is reflected by the mirrors creating a Bokeh image on a screen located on the optical axis at a distance $>2 f$. The Bokeh image creates a compact group of spots on the screen, allowing for fine-tuning of the mirror alignment. The alignment setup, along with images of the $2 f$ and Bokeh alignment spots, are shown in Figure 5.

\section{Pointing of the FAST telescope}

The alignment of the telescope axis is of vital importance to the operation of the telescope. We use the laser (mentioned in section 3) to define the optical axis of the telescope. An astronomical camera with a fast f-number and an angular FOV of approximately $15^{\circ}$ is located on the back cover of the FAST camera as close to the optical axis as possible. A screen is placed far from the telescope along the optical axis (the laser spot is located on the screen). The center of the image of the astronomical camera is overlapped with the laser light spot on the screen (the astronomical camera holder can be tilted to align its optical axis with that of the FAST telescope). The distance between the screen and the telescope defines the angular uncertainty in the alignment of the FAST telescope axis. The misalignment is negligible $<0.01^{\circ}$ for telescope-screen distances $>200 \mathrm{~m}$. Once the alignment of the optical axis of the astronomical camera is complete, an image of the night sky can by obtained. We use the astrometry . net software package to calculate the right ascension and declination of the image center. The result can be transformed into the azimuth and elevation of the FAST telescope. The uncertainty in this method is very small, typically a few arcseconds.

\section{Summary and Future Plans}

We have presented a novel concept for a next-generation lensless Schmidt fluorescence telescope, which features just a few pixels covering a large field-of-view. The first full-scale FAST telescope prototype was installed in October 2016 at the Telescope Array site in central Utah, USA. The prototype was tested using observations of a UV LED flasher, as well as a distant vertical UV laser beam. It has also been used to detect UHECR events in time-coincidence with the Telescope Array fluorescence detector. A second prototype will be installed in October 2017 to increase the angular coverage and allow for the detection of more UHECR events.

\section{Acknowledgements}

This work was supported by the Japan Society for the Promotion of Science through the Grantin-Aid for Young Scientist (A) 15H05443, Grant-in-Aid for JSPS Research Fellow 16J04564 and JSPS Fellowships H25-339, H28-4564. This work was partially carried out by the joint research 


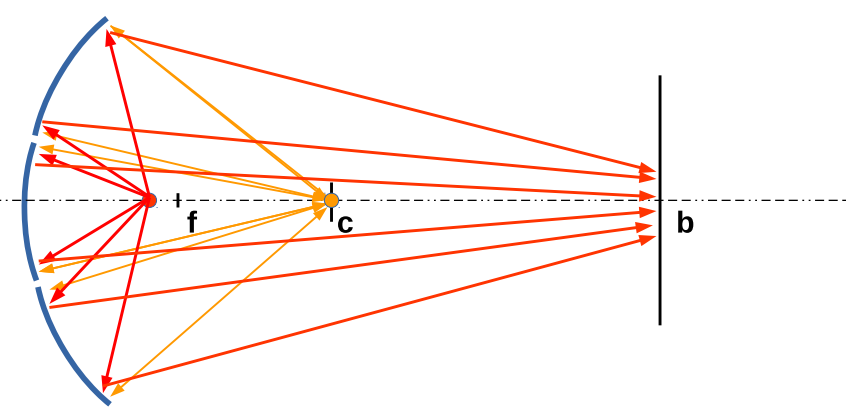

(a) The mirror alignment setup. Two light sources are used. Red for Bokeh and yellow for $2 f$ alignment.

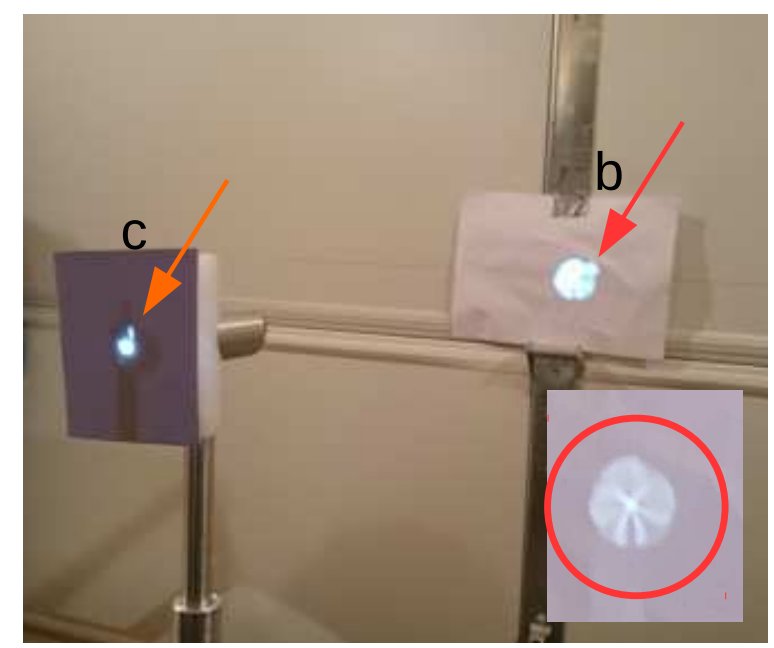

(b) The Bokeh screen (right) showing the alignment procedure. The (left) screen with a built-in light source is used for $2 f$ alignment. $f$ is the focal plane position, $c$ radius of curvature of the mirror segments and $b$ is the location of Bokeh screen.

Figure 5: Mirror alignment, the misaligned mirror segment is visible on both screens - top right close to the spot center (see the arrow). The detail in the Bokeh of the aligned mirror is shown in the red circle (right bottom).

program of the Institute for Cosmic Ray Research (ICRR), University of Tokyo. This work was supported in part by NSF grant PHY-1412261 and by the Kavli Institute for Cosmological Physics at the University of Chicago through grant NSF PHY-1125897 and an endowment from the Kavli Foundation and its founder Fred Kavli. The Czech authors gratefully acknowledge the support of the Ministry of Education, Youth and Sports of the Czech Republic project No. LG15014, LE13012, LO1305, LM2015038, LTAUSA17078, EU/MSMT CZ.02.1.010.00.016_0130001402.

\section{References}

[1] T. Fujii et al., Detection of ultra-high energy cosmic ray showers with a single-pixel fluorescence telescope, Astropart. Phys. 74 (2016) 64-72, [1504.00692].

[2] P. Sommers, Capabilities of a giant hybrid air shower detector, Astropart.Phys. 3 (1995) 349-360. 
[3] Pierre Auger Collaboration, J. Abraham et al., The Fluorescence Detector of the Pierre Auger Observatory, Nucl.Instrum.Meth. A620 (2010) 227-251, [0907 . 4282].

[4] Telescope Array Collaboration, M. Fukushima et al., Telescope array project for extremely high energy cosmic rays, Prog.Theor.Phys.Suppl. 151 (2003) 206-210. 